\title{
Los Mensajes Híbridos en el marketing postmoderno: una propuesta de taxonomía
}

\section{Hybrid Messages in postmodern marketing: a taxonomical proposal}

\author{
Joaquín de Aguilera Moyano \\ Profesor asociado y director académico de la especialidad \\ Integrated Marketing Communications \\ (Instituto de Empresa Business School. España)
}

\begin{abstract}
Miguel Baños González
Profesor Titular Departamento: Ciencias de la Comunicación y Sociología (Universidad Rey Juan Carlos)

Francisco Javier Ramírez Perdiguero

Decano y profesor de Pensamiento Creativo e Innovación

(ISEAD Business School. España)
\end{abstract}

Fecha de recepción: 8 de julio de 2015

Fecha de revisión: 1 de febrero de 2016

Para citar este artículo: Aguilera Moyano, J. de, Baños González, M. y Ramírez Perdiguero, F. J. (2016): Los Mensajes Híbridos en el marketing postmoderno: una propuesta de taxonomía, Icono 14, volumen (14), pp. 26-57. doi: 10.7195/ri14.v14i1.890 
27 | Joaquín de Aguilera Moyano, Miguel Baños González y Francisco Javier Ramírez Perdiguero

INNOVACIÓN TEÓRICA

\section{Resumen}

Podemos entender un "mensaje híbrido" como todo intento pagado de influenciar a las audiencias para un objetivo comercial, utilizando comunicaciones que proyectan un carácter no-comercial (Balasubramanian, 1994). Durante las dos décadas transcurridas, los mensajes híbridos han crecido en importancia y presencia. Algunos de estos mensajes, como el patrocinio o el emplazamiento de producto, han sido ampliamente estudiados, no así formas más novedosas como el branded content, el content marketing o el branded entertainment. No existe consenso entre los diferentes autores sobre el alcance, naturaleza, definición y clasificación de estas nuevas formas de comunicación. En este trabajo se ha realizado una exhaustiva revisión de las publicaciones aparecidas en los últimos años, lo que ha permitido clarificar los tres conceptos aludidos, así como realizar una propuesta de clasificación de los mensajes híbridos, en la que el principal factor de diferenciación es la intencionalidad en la creación del contenido.

Palabras clave: Mensajes híbridos - Comunicaciones de marketing - Branded content - Content marketing - Branded entertainment - Engagement

\section{Abstract}

Hybrid messages may be defined, according to Balasubramanian (1994), as any paid attempt of influencing an audience with a commercial goal, by using communications with non-commercial appearance. During the last two decades, hybrid messages have grown in both importance and presence. Some types of hybrid messages, as sponsorship or product placement, have been widely studied, which is not the case of more novel manifestations, as could be branded content, content marketing or branded entertainment. There is no a common agreement on the nature, reach, definition and classification of those new ways of communication. A comprehensive review of published works on the subject has been carried for this paper, from which a clarification of all three concepts has been made, as well as a proposal of classification, whose main differentiating factor is the intention in content creation.

Key Words: Hybrid messages - Marketing communications - Branded content Content marketing - Branded entertainment - Engagement 
Los Mensajes Híbridos en el marketing postmoderno: una propuesta de taxonomía | 28

INNOVACIÓN TEÓRICA

\section{Introducción}

Algunos de los más destacados estudiosos y profesionales del marketing coinciden en señalar, desde hace tiempo, que el marketing tradicional ha muerto (Zyman, 1999; Kotler, 2005; Keller, 2008). Si bien es cierto que los cambios se han producido en todos los ámbitos (mercado, ecosistema de medios, consumidor, sociocultural y económico), la revolución tecnológica y el propio aprendizaje de los consumidores han provocado cambios profundos en el mundo de los negocios y han dotado al público de un poder que ha revolucionado las relaciones entre las marcas y sus clientes.

El nuevo paradigma emergente de marketing (customer centric, relacional, experiencial, pull...), cuyo objetivo es establecer relaciones duraderas entre el consumidor y la marca, mediante el ajuste de las experiencias propuestas por la marca con las deseadas por el consumidor, se enfrenta a un consumidor empoderado como nunca antes lo ha estado. Un consumidor que busca establecer relaciones con las marcas que le generen experiencias positivas y le proporcionen valor, en cuya creación quiere desempeñar un papel activo; quiere establecer con ellas un diálogo transparente y fluido, en el que no le presionen con sus mensajes.

En este nuevo escenario de marketing la búsqueda de consumer engagement desempeña un papel clave en el marketing centrado en el consumidor. Se le ve como una fuerza motriz fundamental detrás del comportamiento y de la toma de decisiones del consumidor posmoderno (Gambetti y Graffigna, 2010).

Este nuevo modo de hacer marketing, junto a los nuevos hábitos de consumo de medios, conllevan inseparablemente una nueva forma de comunicarse con los consumidores, en la que las herramientas tradicionales pierden eficacia y eficiencia, dando paso a otras que respetan la libertad de acceso, consumo y diálogo de las audiencias.

La industria publicitaria se encuentra en un punto de inflexión, pasando del modelo tradicional de interrupción y repetición en medios de masas a una familia de modelos publicitarios centrados en la relevancia (Rappaport, 2007). 
Los consumidores demuestran una creciente falta de tolerancia hacia los mensajes de marketing que son irrelevantes para sus vidas o que no han solicitado. Es pues la relevancia del mensaje el factor clave para captar la atención de los consumidores (Martí, 2010). Un mensaje es relevante cuando aporta valor añadido, en forma de información y/o de entretenimiento.

El nuevo modelo consiste en la creación sistemática de contenidos y mensajes que sean únicos, bien ejecutados y relevantes, de tal manera que su resonancia sea en los consumidores más profunda y significativa que antes. En lugar de interrumpir a los consumidores con anuncios, se trata de atraerles, de engancharles (engage), con utilidad, entretenimiento, información relevante o cualquier otro tipo de contenido valioso, que sea verdaderamente bien acogido por el consumidor (Mayar y Ramsey, 2011). Frente a la tradicional comunicación de tipo push, se impone otra de tipo pull: atracción, conocimiento mutuo y diálogo (Ramos, 2006).

Estos contenidos deben ser capaces de incorporar el mensaje comercial sin que resulte intrusivo. Es vital encontrar un equilibrio entre la marca (su identificación) y el contenido (la presentación de información o entretenimiento que proporciona al consumidor una experiencia positiva).

Los mensajes que mejor se adaptan a estos requisitos son los conocidos como mensajes híbridos.

\section{Métodos}

\section{Objetivos}

El objetivo general de este trabajo es clarificar la situación de los mensajes híbridos entre los que actualmente existe una gran confusión, dada la variedad de técnicas y tipos de mensajes que se integran en esta categoría. Para conseguirlo establecemos los siguientes objetivos específicos:

- Delimitar la naturaleza y alcance de diferentes técnicas que se integran dentro de lo que se conoce como mensajes híbridos. 
Los Mensajes Híbridos en el marketing postmoderno: una propuesta de taxonomía | 30

- Definir algunas de las técnicas más novedosas -es decir, aquellas técnicas que están teniendo en los últimos tiempos un uso relevante (entre las que destacan las diversas técnicas de branded content), en términos del crecimiento de su participación en las inversiones de comunicaciones de marketing, frente a otras que, por oposición, se consideran tradicionales (entre las que destaca la publicidad intrusiva o, entre los mensajes híbridos, el emplazamiento de producto y el patrocinio)- consideradas mensajes híbridos, sobre las que existe una gran confusión: advergaming, advertainment, branded content, branded entertainment y content marketing.

- Establecer una clasificación de los mensajes híbridos atendiendo a criterios objetivos; siendo el principal de ellos, como se verá más adelante, la intencionalidad en la creación del contenido. Dentro de la clasificación de las formas de branded content, se utilizarán criterios como el objeto del contenido y las plataformas utilizadas para su difusión, mientras que entre el resto de mensajes híbridos, el principal criterio de clasificación es su nivel de integración.

\section{Metodología}

Como ya hemos comentado, los principales fenómenos estudiados como mensajes híbridos son, en la mayoría de los casos, con las importantes excepciones del patrocinio y del emplazamiento de producto, muy novedosos.

Algunos de ellos han tenido cierta presencia anterior -como el advertaiment o el advergaming, primeras menciones a la combinación de un mensaje publicitario con entretenimiento y videojuegos respectivamente- pero en sus manifestaciones más avanzadas -branded content, content marketing y branded entertainment- son fenómenos que han ganado su enorme importancia en tiempos más recientes, a partir sobre todo de la universalización del uso de Internet.

Por ejemplo, es el lanzamiento por la compañía de automoción BMW de la serie de episodios online The Hire el que se considera como el nacimiento del branded entertainment moderno. Esta novedad de las nuevas fórmulas de comunicación 


\section{INNOVACIÓN TEÓRICA}

implica que estén todavía insuficientemente estudiados y que, por consiguiente, exista cierta confusión conceptual y taxonómica al respecto.

Por todo ello, los pasos que hemos dado para obtener y analizar las diferentes fuentes documentales existentes sobre los fenómenos de branded content, content marketing y branded entertainment, ha sido el de explorar dos agregadores de bases de datos:

1. La biblioteca virtual del Instituto de Empresa Business School, que realiza la búsqueda a través de las siguientes bases de datos académicas:

- ABI/INFORM Global

- Business Source Complete

- Ebrary

- Emerald Group Publishing Limited

- IEEE Publications Database

- JSTOR Arts \& Sciences I Collection

- JSTOR Arts \& Sciences II Collection

- JSTOR Business I Collection

- JSTOR Mathematics \& Statistics Collection

- SAGE Knowledge

- ScienceDirect

- Taylor and Francis Journals

- WorldCat.org

2. Google Academic

En ambos cosas se realizó la búsqueda de fuentes publicadas desde el 1 de enero de 2008 hasta el 31 de diciembre de 2013, que incluyesen en su título las expresiones branded content, content marketing $\mathrm{y} / \mathrm{o}$ branded entertainment.

Dada la novedad de los fenómenos estudiados y, por consiguiente, la todavía escasa producción escrita sobre ellos, se buscaron también en la librería 
Los Mensajes Híbridos en el marketing postmoderno: una propuesta de taxonomía | 32

INNOVACIÓN TEÓRICA

online www.amazon.com aquellos libros que incluyesen en su título las antedichas expresiones, con un resultado muy magro.

Por último, se recurrió a las organizaciones profesionales vinculadas a las actividades de branded content, content marketing y branded entertainment, cuya búsqueda se realizó a través de Google. También se revisaron festivales y acontecimientos relacionados con dichas actividades, como la sección dedicada a "Branded Content \& Entertainment" del Festival de Cannes.

Pero para realizar un correcto y completo ejercicio taxonómico sobre los mensajes híbridos no es suficiente con un exhaustivo análisis de las fuentes existentes sobre sus más modernas manifestaciones; es preciso también analizar la naturaleza y revisar las definiciones de las manifestaciones de mensajes híbridos más tradicionales, como los citados patrocinio y emplazamiento de producto, que han sido más ampliamente estudiadas. Para ellas hemos recurrido a fuentes más clásicas, así como a explorar los anteriormente citados agregadores/buscadores en busca de fuentes más actuales.

Partiendo de esta situación, hemos definido los elementos diferenciadores de los diversos tipos de mensajes híbridos, lo que nos ha permitido elaborar una clasificación de los mismos y la definición de algunos tipos de mensajes que, como el branded content, content marketing y branded entertainment, no estaban definidos con precisión.

\section{Desarrollo}

\subsection{Mensajes híbridos}

El concepto de mensaje híbrido fue desarrollado por Balasubramanian en 1994, siendo hoy aceptado por la inmensa mayoría de los autores consultados (Baños y Rodríguez, 2003; Hudson y Hudson, 2006; Ramos, 2006; Russell, 2007; Lehu, 2007; Tuomi, 2010; Martí, 2010; Walton, 2010; Regueira, 2012) y es de uso común en la literatura sobre comunicaciones de marketing para designar un tipo de mensajes que abarca una amplia gama de variedades.

DOI: ri14.v14i1.890 | ISSN: 1697-8293 | Año 2016 Volumen 14 N$^{\circ} 1$ | ICONO14 


\section{INNOVACIÓN TEÓRICA}

Según Balasubramanian (1994), cuando las organizaciones pretenden exponer a las audiencias a información relacionada con un producto recurren con frecuencia a dos herramientas de comunicación: publicidad y publicity. La primera se refiere a las comunicaciones por cuya difusión se paga, y que identifican claramente a quien lo hace, mientras que la segunda designa los mensajes por los que no se paga y cuyo emisor no se identifica.

Dos son los elementos básicos de la comunicación que Balasubramanian considera esenciales en su teoría:

- el mensaje.

- la fuente percibida.

Lo ideal es encontrar un tipo de mensaje que permita, al mismo tiempo, que el emisor controle el mensaje y que la audiencia lo perciba como creíble. Los mensajes híbridos son los que combinan ambas virtudes. Balasubramanian (1994) los define como: todo intento pagado de influenciar a las audiencias para un objetivo comercial, utilizando comunicaciones que proyectan un carácter no-comercial; bajo estas circunstancias lo más probable es que las audiencias no sean conscientes del intento de influencia comercial y que procesen, por tanto, tales comunicaciones de manera diferente a como lo harían con un mensaje comercial.

Una interpretación posmoderna del concepto permite identificar a los mensajes híbridos como aquellos que, de una u otra manera, combinan un mensaje comercial (favorable a una marca) con contenido no-comercial (no enfocado a una propuesta de compra y que proporciona a la audiencia una experiencia positiva).

Balasubramanian identifica algunos de los factores claves en esta materia:

- El grado de control sobre el mensaje y el formato.

- Lo explícito o implícito de la presencia de la marca.

- El consiguiente grado de integración de la marca en la historia. 
Los Mensajes Híbridos en el marketing postmoderno: una propuesta de taxonomía | 34

INNOVACIÓN TEÓRICA

Aunque al utilizar los medios para transmitir mensajes las organizaciones deben ceder parte del control sobre el mensaje, está comúnmente aceptado que el mensaje se percibe como más creíble al ser diseminado por los medios en lugar de por las propias organizaciones (publicidad) (Walton, 2010).

Según señala Tuomi (2010), basándose en el modelo de Nebenzahl y Jaffe de 1998, existen además otros dos elementos a considerar en las comunicaciones de marketing:

- Disfraz: grado en el que el emisor puede ser identificado y/o la capacidad para percibir si el mensaje es publicidad pagada o no.

- Prominencia: grado en el que el mensaje es más o menos secundario con respecto a una comunicación destacada.

Desde el punto de vista de las comunicaciones de marketing, el mensaje ideal sería aquel que tuviese altos niveles tanto de Disfraz como de Prominencia; sin embargo, la publicidad tradicional cuenta con bajos niveles de ambos.

\subsubsection{Elementos diferenciadores en la estructura}

Podemos realizar una primera aproximación a una clasificación de los mensajes híbridos a partir de una serie de elementos diferenciadores en su estructura.

\section{Implícito versus explícito}

Según D'Astous y Séguin (1999), en un trabajo centrado en el patrocinio de televisión aplicable a otros tipos de mensaje híbrido (Hudson y Hudson, 2006; Tuomi, 2010) el principal elemento de diferenciación es su carácter de implícito o de explícito.

En un mensaje implícito las marcas desempeñan un papel contextual pasivo, la presencia de la marca no está expresada formalmente y los beneficios del producto no están claramente expuestos. En los mensajes explícitos las marcas desempeñan un papel activo, estando formalmente expresadas en el programa y cuyos atributos están claramente expuestos. 


\section{INNOVACIÓN TEÓRICA}

Dentro de los mensajes explícitos se distingue entre integrados (están incluidos dentro del contenido) y no-integrados (la marca está formalmente expresada pero no están incluidos dentro del programa). El patrocinio en televisión sería un claro caso de explícito no-integrado: la marca está explicitada pero no aparece dentro del contenido del programa, sino inmediatamente antes o después del mismo.

\section{Nivel de control y de integración}

El nivel de control se refiere a la capacidad del anunciante de influir en el contexto en que va a aparecer la marca; por su parte, el nivel de integración denota el nivel de conexión con la trama. Generalmente, a mayor nivel de integración, mayor nivel de control.

Sheehan y Guo (2005) dividen estos mensajes en cuatro tipos, a partir de ambas dimensiones:

1. Tradicional: productos o servicios habitualmente utilizados como atrezzo o props; el nivel de integración en la narrativa es bajo $\mathrm{y}$, al desempeñar un papel menor, el nivel de control también es bajo. El mensaje de marca está disimulado y es secundario con respecto a la trama.

2. Ampliado: las marcas adquieren una mayor conexión con la trama. Su nivel de integración sigue siendo bajo (pueden sustituirse por productos similares), aunque pueden utilizarse recursos auditivos y visuales.

3. Integrado: son parte integral de la trama en una conexión a largo plazo.

4. Asimilado: aquellos casos en que la marca se convierte en el eje de la trama o del programa.

Algunos autores consideran la integración de producto como un tipo de mensaje per se, diferente a otros tipos como, por ejemplo, el emplazamiento de producto; para Russell (2007) la integración de producto se refiere a un emplazamiento más extenso en el que el producto realmente desempeña un papel en la trama de un episodio. 
Los Mensajes Híbridos en el marketing postmoderno: una propuesta de taxonomía | 36

INNOVACIÓN TEÓRICA

\subsubsection{Tipos de Mensajes Híbridos}

Balasubramanian identifica varios tipos de mensajes híbridos "establecidos":

1. Emplazamiento de Producto: mensaje pagado dirigido a influenciar a las audiencias mediante la planificada y poco prominente introducción de un producto con marca en una película (o programa de televisión).

2. Programa con acuerdo (tie in): mensaje pagado porque es un arreglo de intercambio entre un programa y una marca. Habitualmente este acuerdo implica la compra de espacio publicitario en el programa.

3. Anuncio con la duración de un programa: mensaje de producto pagado emitido a las audiencias de televisión, utilizando un formato que se parezca a un auténtico programa tanto en contenido como en duración.

Así como otro grupo de mensajes a los que califica de "emergentes":

1. Arte enmascarado: cualquier obra de arte (pintura, escultura, música, teatro, literatura) que presenta productos con marca con un objetivo comercial deliberado, aunque no siempre obvio.

2. Noticias enmascaradas: mensaje híbrido insertado entre fuentes de noticias.

3. Portavoz enmascarado: dos posibles tipos:

- Experto enmascarado: una persona, desempeñando el papel de experto, para acentuar la credibilidad.

- Famoso enmascarado: oculta su papel de portavoz pagado para promocionar marcas durante sus apariciones públicas.

Con la revolución digital surgen además nuevas fórmulas, como la de los "influenciadores" (influencers), escritores de blogs con credibilidad, a los que en ocasiones se paga (con dinero o en especies) para que recomienden una determinada marca dentro de su área de influencia (experto enmascarado). 
37 | Joaquín de Aguilera Moyano, Miguel Baños González y Francisco Javier Ramírez Perdiguero INNOVACIÓN TEÓRICA

A los tipos de mensajes híbridos que identifica Balasubramanian en su trabajo original se han unido otros muchos a los largo de estos años:

- Advergaming

- Advertainment

- Branded content

- Branded entertainment

- Content marketing

- Custom publishing

Aunque algunas de estas técnicas de mensaje híbrido tienen bastante antigüedad y sobre algunas de ellas se han publicado numerosos trabajos, otras han alcanzado su esplendor en épocas más recientes, a partir sobre todo del auge de Internet y de los nuevos modos de comunicación audiovisual y digital.

No existe una distinción clara, ni en el terreno académico ni en el profesional, entre los diferentes conceptos, ni existe por tanto una taxonomía mayoritariamente aceptada.

Algunos autores, por ejemplo, consideran al emplazamiento de producto prácticamente como sinónimo de mensaje híbrido y a otras técnicas como versiones y/o herramientas de este. Así, para Lehu (2007) emplazamiento de producto y emplazamiento de marca tienen un mismo significado y entiende el branded entertainment como una versión, más sofisticada, del emplazamiento de producto, cuando se produce en el mundo del entretenimiento. Williams, Petrosky, Hernández y Page (2011), consideran al emplazamiento de producto como sinónimo de emplazamiento de marca, patrocinio dentro de un programa, branded entertainment 0 integración de producto. Hudson y Hudson (2006), Tuomi (2010) y Soares (2011) consideran el branded entertainment y el advertainment como extensiones o versiones mejoradas, más maduras y sofisticadas, del emplazamiento de producto.

Otros autores, por el contrario, consideran el emplazamiento de producto como un tipo de advertainment, al igual que lo serían el branded entertainment y el branded content que, para Russell (2007), tendrían ambos el mismo significado. 
Los Mensajes Híbridos en el marketing postmoderno: una propuesta de taxonomía | 38

Un tercer grupo de autores distinguen claramente entre el emplazamiento de producto y el advertaiment, branded content o branded entertainment, a los que consideran sinónimos, entendiendo el primero como la integración, en mayor o menor grado, de una marca/producto dentro de un contenido no comercial, y a los tres últimos como el desarrollo de contenidos propios desde la marca (Pino, y Olivares, 2007; Ramos, 2006; Regueira, 2012; Canter, Asmussen, Michels, Butler y Thomson, 2013; Valiquette, 2013; Neurads, 2013).

Un grupo de autores engloba todos aquellos mensajes comerciales en televisión, que sean diferentes a los empleados en los tradicionales spots, dentro del concepto de "nuevas formas de publicidad" (Reinares y Reinares, 2013).

Un último grupo de autores da preeminencia al content marketing como concepto principal, del que emanan el resto (Rose y Pulizzi, 2011; Pulizzi, 2012; Rahim y Clemens, 2012; Duncan, 2012; Ramos, 2013).

Como puede observarse, el nivel de confusión entre los citados conceptos es obvio.

Tampoco proporcionan definiciones claras sobre estos conceptos las diferentes asociaciones profesionales: la Branded Content Marketing Association, The Content Council, la Content Marketing Association o la Entertainment and Resources Marketing Association.

Esta situación demanda una clarificación y clasificación de los mensajes híbridos, para lo cual partimos de una serie de premisas:

- Considerar el emplazamiento de producto y el emplazamiento de marca como una misma técnica: la inclusión de una marca dentro de un contenido no centrado en la marca.

- Considerar el advertainment y el branded entertainment como una misma técnica: la creación de contenidos de entretenimiento propios de la marca. 
39 | Joaquín de Aguilera Moyano, Miguel Baños González y Francisco Javier Ramírez Perdiguero INNOVACIÓN TEÓRICA

- Considerar el advergaming como un tipo de branded entertainment: creación de contenidos propios de la marca en videojuegos.

- Considerar el custom publishing como un tipo de content marketing: la creación de publicaciones alrededor de una marca.

\subsection{Branded content}

Ya hemos visto la enorme confusión que existe entre los diferentes conceptos, pero incluso entre aquellos que defienden que el branded content es la expresión más sofisticada y moderna del emplazamiento de producto, existe una concepción de él coincidente con la del resto de autores: el branded content/entertainment consiste en la creación por la marca de sus propios contenidos. Así, para Lehu (2007) el branded entertainment debería representar para la marca una oportunidad de escribir verdaderas historias, integrando su identidad y su personalidad; con esta técnica, la marca se embarca en la producción de sus propios programas para controlar perfectamente la integración. Tuomi (2010) añade que el contenido puede ser completamente producido, financiado y controlado por una marca. Martí (2010) considera equivalentes el branded content y el branded entertainment definiéndolo básicamente como contenido de entretenimiento que se genera en torno a una marca para alcanzar los objetivos de marketing establecidos.

Lo mismo ocurre en el sector profesional, que entienden el branded content como una herramienta cuyo fin es el de proporcionar contenido relevante, por útil o por entretenido, que atraiga y mantenga la atención de sus públicos objetivos, sorteando su resistencia natural a la publicidad; bajo la premisa de que una experiencia positiva con el contenido generará sentimientos positivos hacia la marca que lo ha hecho posible (Duncan, 2012).

La plataforma online Neurads (2013) ha elaborado una lista de diferencias entre branded content y emplazamiento de producto (tabla 1), que puede resultarnos muy útil para nuestro propósito: 
Los Mensajes Híbridos en el marketing postmoderno: una propuesta de taxonomía | 40 INNOVACIÓN TEÓRICA

\begin{tabular}{|c|c|}
\hline Branded Content & Emplazamiento \\
\hline Es contenido & $\begin{array}{c}\text { Es una técnica de comunicación de marca dentro } \\
\text { de OTRO CONTENID0, no contenido en sí mismo }\end{array}$ \\
\hline Refleja los valores de marca & $\begin{array}{c}\text { Se integra en contenidos que pueden no tener } \\
\text { nada que ver con los valores de marca }\end{array}$ \\
\hline Elemento activo & $\begin{array}{c}\text { La marca es un elemento más dentro de } \\
\text { contenido independiente. Elemento pasivo }\end{array}$ \\
\hline La marca tiene el control sobre cómo se \\
transmite el mensaje & $\begin{array}{c}\text { El control de la marca está limitado } \\
\text { otras se impone }\end{array}$ \\
\hline Suele incluir emplazamiento de producto & En ocasiones se integra con naturalidad; \\
\hline
\end{tabular}

Tabla 1: Diferencias entre branded content y emplazamiento de producto (Neurads, 2013)

Canter et al. (2013) ilustran de la siguiente manera la perspectiva sobre el branded content de lo que llaman Trade Mark Owner, TMO (que podemos traducir como Propietario o Titular de la Marca Registrada):

1. El branded content es iniciado por el TMO, digitalmente o no digitalmente.

2. El nivel de control del TMO va de alto (programa de televisión) a bajo (tweet).

3. Suele estar financiado por el TMO.

4. Sus principales objetivos son:

- Crear un significado o manifestaciones de marca positivos entre los stakeholders

- Conectar y engage con determinados stakeholders

5. La estrategia puede ser:

- Entretenimiento

- Informativa

- Educativa

- Útil (cumplir una determinada función)

DOI: ri14.v14i1.890 | ISSN: 1697-8293 | Año 2016 Volumen 14 N$^{\circ} 1$ | ICONO14 
41 | Joaquín de Aguilera Moyano, Miguel Baños González y Francisco Javier Ramírez Perdiguero INNOVACIÓN TEÓRICA

6. Más pull que push.

7. Predominantemente engagement frente a interrupción.

8. Potencialmente omnicanal.

La conclusión que claramente se desprende de todas las definiciones y reflexiones anteriores es que el branded content es contenido generado a partir de una marca.

Resulta igualmente obvio que una de las principales características del patrocinio y del emplazamiento de producto es la de no ser una parte esencial del contenido, sino algo extraño a este y en el que se inserta, con mayor o menor grado de integración.

En definitiva, lo que esencialmente distingue al branded content del patrocinio $\mathrm{y}$ del product placement es la intencionalidad en la creación del contenido que vehicula el mensaje de marca.

En el último caso, el contenido tiene una intención absolutamente ajena a la de transmitir un mensaje de marca. La intención del creador/productor es que el contenido sea el fin en sí mismo.

Mientras que en el branded content, la intención al crear el contenido es transmitir un mensaje de marca, de manera más o menos explícita. La intención del creador/productor en este caso es que el contenido sea un medio para el fin de transmitir el mensaje de la marca.

Por tanto, defendemos que es la intencionalidad de la creación del contenido el primer y principal factor de diferenciación de los mensajes híbridos, clasificándolos en dos grandes grupos: aquellos en que el mensaje de marca no forma parte del contenido original, sino que se inserta en él; y aquellos en los que el mensaje de marca es la esencia del contenido original. 
Los Mensajes Híbridos en el marketing postmoderno: una propuesta de taxonomía | 42 INNOVACIÓN TEÓRICA

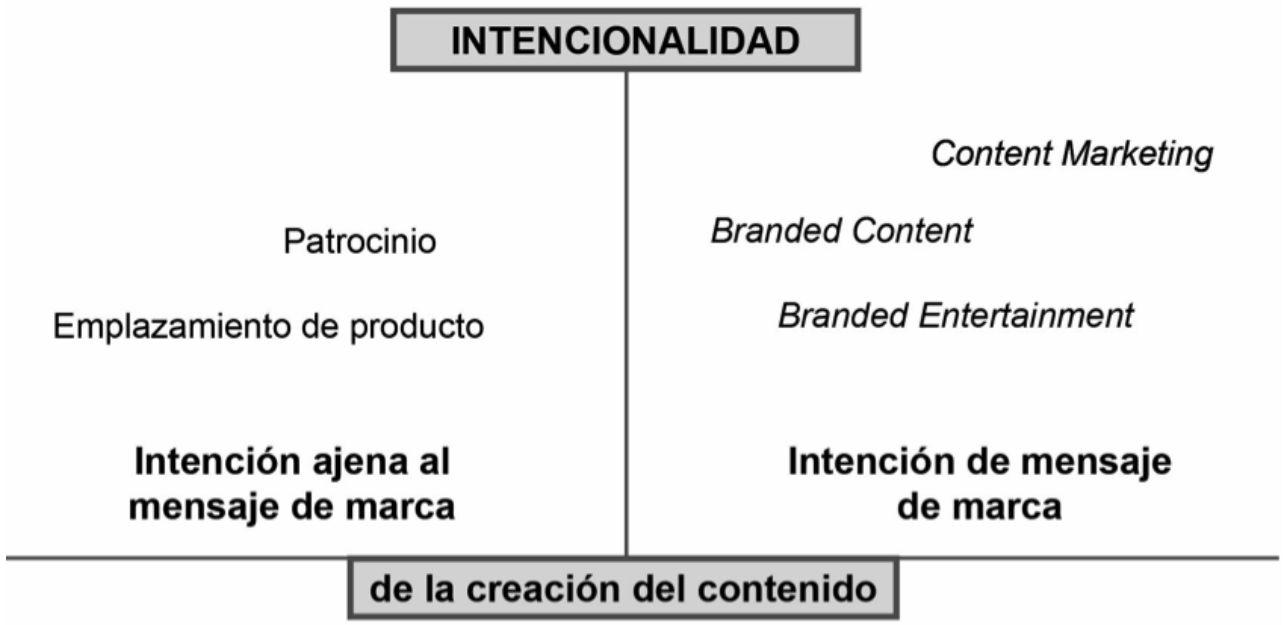

Figura 1: Clasificación según la intencionalidad del contenido (Fuente: Aguilera, 2014)

Nuestra siguiente propuesta es que el branded entertainment (y su sinónimo advertainment) y el content marketing son dos sub-formas de branded content.

\subsubsection{Content marketing}

A diferencia de lo que ocurre con otros tipos de mensajes híbridos, parece existir un amplio consenso sobre lo que significa el content marketing.

Hay que hacer notar que la mayoría de los autores que han tratado este concepto proceden del ámbito profesional, siendo muy escasos todavía los que lo han hecho desde el ámbito académico.

El Content Marketing Institute (2012), principal centro impulsor y divulgador del content marketing, define a este como una técnica de marketing que consiste en la creación y distribución de contenido relevante y valioso para atraer, adquirir y engage a una claramente definida y estudiada audiencia objetivo, con el fin de promover una acción provechosa por parte del cliente.

Pulizzi (2012), creador del citado instituto, refuerza la idea anterior, pero subraya el hecho de que es la propia marca quien crea el contenido. 
43 | Joaquín de Aguilera Moyano, Miguel Baños González y Francisco Javier Ramírez Perdiguero

\section{INNOVACIÓN TEÓRICA}

En la misma línea se manifiestan la mayoría de las fuentes consultadas, aunque varios de ellos añaden algún detalle de valor a la definición. Así, Duncan (2012) señala que el alcance del content marketing llega hasta el paso anterior al de realizar una propuesta de venta o una llamada a la acción; Zmuda (2013) recuerda que se transmite vía medios propios y ganados, nunca pagados, pues en este caso sería publicidad, opinión que comparten plenamente Lieb, Silva y Tran (2013); Nosrati, Karimi, Mohamadi y Maleikian (2013) lo circunscriben a medios y publicaciones; y Ramos (2013), finalmente, incluye un nuevo objetivo, el de educar, junto a los ya citados de engage y adquirir al cliente o prospecto.

Por lo tanto, podríamos redactar la siguiente definición "de consenso": una técnica de marketing que consiste en la creación y distribución, por parte de una marca, de contenido relevante, valioso y convincente, a través de medios propios y/o ganados, para atraer, adquirir, educar y engage a clientes y prospectos; el contenido puede ser informativo $\mathrm{y} / \mathrm{o}$ educativo $\mathrm{y}$, si bien no realiza ninguna propuesta de venta o llamada a la acción, tiene como objetivo el de promover una acción beneficiosa para la marca por parte del cliente y/o prospecto.

El content marketing se centra en crear experiencias de valor. Se trata de valiosas piezas de información que enriquecen a la comunidad y posicionan a la marca como líder en su campo; contenido que se puede compartir de manera inmediata $\mathrm{y}$, sobre todo, focalizado en ayudar a los clientes a descubrir (por sí mismos) que el producto o servicio es el que resuelve su necesidad.

Parece comúnmente aceptado que el primer caso de content marketing -como técnica de marketing- se remonta al lanzamiento, en 1895, de la revista The Furrow (El surco), publicada por el fabricante de tractores John Deere, y que todavía se mantiene en circulación. Su objetivo no era vender directamente productos John Deere, sino proporcionar a los agricultores información sobre nuevas técnicas agrícolas y formación para que su explotación se volviese más rentable. Así, John Deere se convierte en líder y experto en la materia, y cuando los lectores de la revista necesitan equipamiento agrícola se dirigen naturalmente a quien está posicionado como experto en la materia, John Deere. 
Los Mensajes Híbridos en el marketing postmoderno: una propuesta de taxonomía | 44 INNOVACIÓN TEÓRICA

Otros casos relevantes de los inicios de esta técnica de marketing son:

- La publicación de la Guía Michelin, en 1900 en Francia, ofreciendo información sobre mantenimiento de los coches, así como sobre alojamientos, restaurantes...

- En 1904, la compañía de alimentación estadounidense Jell-0 distribuyó gratuitamente un libro de recetas de cocina, en el que se trata a su producto de postre como un alimento versátil.

Estos antecedentes del content marketing son claros ejemplos de custom publishing (publicaciones de empresa). Por tanto, parece poderse inferir que el content marketing es la versión de la era digital del custom publishing pues puede presentarse, además de como publicaciones, bajo gran variedad de formas: noticias, vídeos, e-books, infografías, estudios de casos, manuales, fotografías... (Nosrati et al., 2013).

Esta nueva forma de distribución de contenido, digital, elimina las barreras que existen para la publicación de contenido en soporte tradicional, impreso, que es compleja y cara; cualquiera, incluidas las marcas, puede hoy publicar contenido digital en cuestión de minutos a coste mínimo.

Esta facilidad de publicación permite que las marcas se estén convirtiendo en "compañías de medios", en editores, pues tienen a su disposición una amplia red de medios propios.

La diferencia entre estas nuevas compañías de medios y las tradicionales estriba en que, en estas últimas, el contenido se crea para que genere ingresos directamente por su venta $\mathrm{y} / \mathrm{o}$ por sus ventas de publicidad; mientras que las primeras crean contenido para obtener ingresos indirectamente, atrayendo y reteniendo clientes (Pulizzi, 2012). Ambas están compitiendo por conseguir y retener la atención de las audiencias.

Además, las marcas buscan la distribución de sus contenidos en medios de terceros, medios ganados, entre los que se encuentran las redes sociales, los agregadores, otras comunidades y las menciones en medios (tarea esta última tradicional de las relaciones públicas). 


\section{INNOVACIÓN TEÓRICA}

El content marketing es educativo por naturaleza (Rahim y Clemens, 2012) y trata de una marca, de lo que la hace única, y de su contexto; y puede utilizarse tanto como herramienta para generar ventas, como herramienta de largo plazo para construir confianza. Ayuda a los consumidores a resolver problemas y les invita a engage con una marca. De esta manera los consumidores intercambian lealtad por conocimiento, sobre todo si las marcas se consolidan como verdaderas expertas en su campo.

Rahim y Clemens (2012) realizaron, en Europa y en Estado Unidos, un estudio entre profesionales del marketing, del que se desprende que el primer objetivo del content marketing es, para ambos colectivos, la generación de leads cualificados.

Rose y Pulizzi (2011) señalan los siguientes objetivos, ordenados según el embudo de compra, como los principales que pueden alcanzarse mediante el uso del content marketing:

- Conocimiento o refuerzo de marca.

- Generación de leads.

- Conversión.

- Servicio al cliente.

- Venta de otros productos.

Y añaden cuatro dimensiones más allá de la captación de clientes: satisfacción, retención, upsell y evangelismo.

Ramos (2013) destaca los siguientes tipos de content marketing:

- Enseñanza sobre el producto o servicio.

- Entretenimiento entendido como informar, educar y vender de manera entretenida, utilizando vídeos, fotos, sonido...

- Herramientas y aplicaciones.

- Agregación de contenido. 
Los Mensajes Híbridos en el marketing postmoderno: una propuesta de taxonomía | 46 INNOVACIÓN TEÓRICA

En cuanto a los canales, señala los siguientes:

- El sitio web corporativo que incorpora los medios propios.

- Redes sociales: medios ganados.

Lieb et al. (2013) elaboran una clasificación de formatos de content marketing: texto, gráficos, audiovisuales y aplicaciones y juegos.

Podemos concluir, que las características que definen al content marketing son:

1. Es contenido creado/producido por la marca (es, por tanto, un tipo de branded content).

2. Es contenido de carácter informativo y educativo (no es entretenimiento sino, en algunos casos, contenido entretenido).

3. La materia del contenido es sobre el producto/servicio, la marca/compañía o la categoría/sector.

4. Es mayoritariamente digital.

5. Se distribuye exclusivamente en medios propios y ganados.

\subsubsection{Branded entertainment}

Es la forma que adopta el branded content cuando es entretenimiento, por oposición al content marketing, que es informativo/educativo; con el que además presenta otras diferencias:

1. La materia del branded entertainment no es el producto, la marca/compañía o la categoría/sector; es entretenimiento que transmite los valores de la marca, su identidad y personalidad y cumple unos objetivos de marketing y/o comunicación. 
47 | Joaquín de Aguilera Moyano, Miguel Baños González y Francisco Javier Ramírez Perdiguero INNOVACIÓN TEÓRICA

2. Puede no haber manifestaciones de ningún tipo de la marca (brand free) y puede también ser vehículo de emplazamiento de producto.

3. No tiene por qué ser predominantemente online, pudiendo adoptar muy diversas formas: audiovisual, audio, musical, gráfica, teatral, deportiva, plástica, cultural... y ser difundido por múltiples canales y plataformas.

Hay empresas que han escogido la producción de entretenimiento como vehículo de transmisión de sus mensajes de marca, aplicando los conocimientos de la industria del entretenimiento a la creación de contenidos propios, en los que la trama o el guión están al servicio de los valores de la marca (Ramos, 2006).

La marca debe integrarse en el mundo del entretenimiento de la forma más natural posible, despertando deseos y emociones por sí misma (Lehu, 2007), de tal forma que la combinación del entretenimiento y del atractivo de las historias se convierta en un elemento esencial para que los consumidores perciban la marca de manera positiva (Pino y Olivares, 2007).

Es la marca quien crea el contenido, a la medida de sus necesidades, pero con la condición de ser competitivo como producto de entretenimiento (Regueira, 2012); debe tener suficiente calidad e interés, y poseer entidad propia para su público objetivo como para que elijan el branded entertainment frente a cualquier otro producto de entretenimiento (Ramos, 2006).

No compartimos el punto de vista taxonómico de ciertos autores, que equiparan el branded entertainment con el branded content o que incluyen en él la integración de marca y el contenido financiado por una marca; menos aún el de quienes lo consideran una modalidad del emplazamiento de producto (Hudson y Hudson, 2006). En el primer caso, porque ya hemos establecido que el branded content puede ser informativo/educativo (content marketing) o entretenimiento (branded entertainment), que son conceptos excluyentes (aunque un contenido informativo pueda ser entretenido y un contenido de entretenimiento pueda transmitir información). Por otra parte, hemos establecido que la principal característica del branded content es la de ser contenidos creados/ 
Los Mensajes Híbridos en el marketing postmoderno: una propuesta de taxonomía | 48

INNOVACIÓN TEÓRICA

producidos por la propia marca, de tal forma que la integración de producto, por muy bien hecha que esté, no deja de ser una marca insertada en un contenido ajeno a esta ( $y$, por tanto, generalmente sustituible por otro producto de su misma categoría).

Estos argumentos nos llevan también a equiparar el branded entertainment con el advertainment ya que cualquiera de las definiciones de advertainment estudiadas coincide plenamente con la de branded entertainment.

El branded entertainment es esencialmente una acción para transmitir los valores de marca, su ADN, y para dotarla de una dimensión emocional (Lehu, 2007). Se trata de la creación de vehículos de entretenimiento en los que la marca se mantiene fiel a sus valores y convive en armonía con el contenido (Tuomi, 2010).

Incluso la pieza de entertainment puede llegar a estar totalmente desprovista de la presencia de la marca o de sus productos limitándose a reflejar la personalidad de la marca; en este caso, lo más probable es tener que recurrir a otras acciones de comunicación, notoriamente en medios propios y ganados, pero ocasionalmente también pagados, que garanticen el vínculo entre la pieza de entretenimiento y la marca. Como señala Tuomi (2010), la naturaleza no repetitiva y no intrusiva del branded entertainment, puede necesitar del apoyo de la publicidad para estimular el interés sobre él, como cualquier otro producto de entretenimiento.

Las acciones de branded entertainment no sólo permiten cumplir los objetivos a corto-plazo (notoriedad, preferencia de marca, intención de compra...), sino que contribuyen en gran medida a la construcción de marca a medio y largo-plazo (Lehu, 2007; Regueira, 2012).

Por tanto, y como resultado del análisis anterior, podemos identificar las siguientes características del branded entertainment:

1. Es una categoría de branded content, la que se refiere a contenido de entretenimiento creado y producido por una marca. 
49 | Joaquín de Aguilera Moyano, Miguel Baños González y Francisco Javier Ramírez Perdiguero INNOVACIÓN TEÓRICA

2. Es contenido de entretenimiento creado "a partir de" la marca, de su ADN, con el objetivo de reflejar sus valores, identidad, personalidad y/o posicionamiento.

3. El contenido debe ser de alta calidad, de manera que resulte interesante y atractivo para las audiencias.

4. Las audiencias deben percibir que en el contenido es un producto de entretenimiento más que un mensaje de marca.

5. Es una herramienta a medio y largo plazo de construcción de marca.

6. Puede también atender a objetivos a corto plazo de la marca.

7. Puede adoptar múltiples formas y utilizar cualquier vehículo de entretenimiento.

8. Una modalidad suficientemente importante como para dotarle de identidad propia es el advergaming, o videojuegos creados y producidos por una marca, tanto con objetivos de construcción de marca como de producto (demostrativo).

9. El contenido puede estar absolutamente desprovisto de presencia, visual y/o auditiva, de la marca.

10. Puede existir emplazamiento de la marca.

11. Puede existir igualmente emplazamiento de marcas y/o productos/servicios ajenos a la marca.

12. En ocasiones es conveniente realizar acciones complementarias que refuercen y garanticen la vinculación del contenido con la marca. 
Los Mensajes Híbridos en el marketing postmoderno: una propuesta de taxonomía | 50

INNOVACIÓN TEÓRICA

Como resultado, proponemos la siguiente definición de branded entertainment: es contenido de entretenimiento, del tipo que sea, creado y producido por una marca, que debe resultar lo suficientemente interesante y atractivo como para que la audiencia objetivo decida consumirlo frente a otros productos de entretenimiento del mismo género o sustitutivos. El contenido ha sido creado con el fin de cumplir objetivos de construcción de marca, así como otros posibles de más corto plazo, y puede estar tanto absolutamente desprovisto de presencia de marca como incorporar esta con distintos niveles de integración.

\subsection{Propuesta de clasificación de los mensajes híbridos}

\subsubsection{La intencionalidad de la creación del contenido}

Como ya hemos dicho, el principal factor de diferenciación es la intencionalidad de la creación del contenido que sirve de vehículo al mensaje de marca.

El contenido se crea con intención de vehicular un mensaje de marca o no se crea con esa intención, no hay opciones intermedias.

Así, tenemos por un lado los mensajes híbridos resultado de incorporar o insertar, con menor o mayor nivel de integración, una marca dentro de un contenido que ha sido creado con fines ajenos al de la marca insertada. Aquí tenemos el patrocinio y el emplazamiento de producto/marca.

Por otro lado tenemos mensajes híbridos cuyo contenido tiene como fin transmitir un mensaje de marca, dotándole de suficiente interés y relevancia como para atraer a la audiencia objetivo. En esta área se sitúan el branded content, el content marketing y el branded entertainment.

Más complejo resulta situar las nuevas formas de publicidad en televisión (NFA) en una u otra categoría. Las telepromociones internas y las cartelas de patrocinio entran claramente dentro de la primera categoría, en la que se encuentran el patrocinio y el emplazamiento. 


\section{INNOVACIÓN TEÓRICA}

Los mini-programas, en nuestra opinión, entran en la segunda categoría pues se trata de contenidos supuestamente interesantes para la audiencia creados con el único fin de cumplir con unos objetivos de marca. La diferencia con el content marketing estriba en su carácter de estrategia push (incluidos en bloques publicitarios intrusivos) así como de utilizar medios pagados, en lugar de propios o ganados.

Las telepromociones externas son contenidos claramente creados con el fin de cumplir un objetivo de marca/producto, que utilizan personajes de un programa real, dentro del contexto de este, para aparentar que son portavoces. Tiene, como el caso anterior, características comunes con el content marketing $\mathrm{y}$, también, las características diferenciales de ser push y en medios pagados.

Por tanto, cartelas de patrocinio y telepromociones internas, entrarían dentro de la categoría de mensajes creados con un objetivo ajeno al de marca; mientras que mini-programas y telepromociones externas entrarían en la de contenidos creados con un objetivo de marca.

\subsubsection{Nivel de integración}

Es el principal factor de diferenciación dentro de la categoría de mensajes híbridos insertados dentro de un contenido ajeno al objetivo de la marca.

Pueden tener un nivel mínimo de integración, como los patrocinios de programas, en los que se limitan a señalar, justo antes de empezar e inmediatamente después de finalizar el programa, el hecho del patrocinio por la marca. Y puede alcanzar muy altos niveles de integración, como es el caso de formar parte de la historia o guión en la que se realizará el emplazamiento, que se reescribe de manera que la marca interprete un papel relevante y el contexto sea consecuente con el mensaje de marca a transmitir. Algunos autores califican este nivel de integración como una modalidad diferente, más evolucionada, de emplazamiento, a la que denominan, precisamente, "integración de marca" (Russell, 2007; Lehu, 2007; Tuomi, 2010). 
Los Mensajes Híbridos en el marketing postmoderno: una propuesta de taxonomía | 52

INNOVACIÓN TEÓRICA

En medio se encuentran modalidades como el emplazamiento de marca pasivo, que consiste en la mera presencia de la marca/producto en la pantalla y/o en el texto.

Por tanto, el nivel de integración dibujaría una línea ascendente, en cuyo nivel más bajo se encontraría el patrocinio y en cuyo nivel superior la integración de marca, ubicándose el emplazamiento en el centro.

A mayor nivel de integración se produce también un mayor nivel de control sobre el contexto en que aparece la marca/producto.

\subsubsection{Información versus entretenimiento}

En lo que respecta a los conceptos ubicados dentro de la categoría de los contenidos creados con el fin de cumplir un objetivo de marca, hemos identificado el branded content como el denominador de la totalidad de la categoría; es decir, aquella en que la marca crea y produce sus propios contenidos.

$\mathrm{Al}$ analizar los conceptos de content marketing y branded entertainment, hemos visto que la principal diferencia entre ambas es el tipo de contenido: en el primer caso es información y en el segundo, entretenimiento.

Esta característica diferenciadora conlleva que, en el caso del content marketing, el contenido sea "sobre o alrededor de" la marca; mientras que en el caso del branded entertainment sea "a partir de" la marca, sin que sea imprescindible tratar de esta, sus productos o su categoría.

\subsubsection{Clasificación}

La siguiente infografía (Figura 2) resume la propuesta de clasificación de los mensajes híbridos, donde el principal criterio de diferenciación es la intencionalidad de la creación del contenido. 


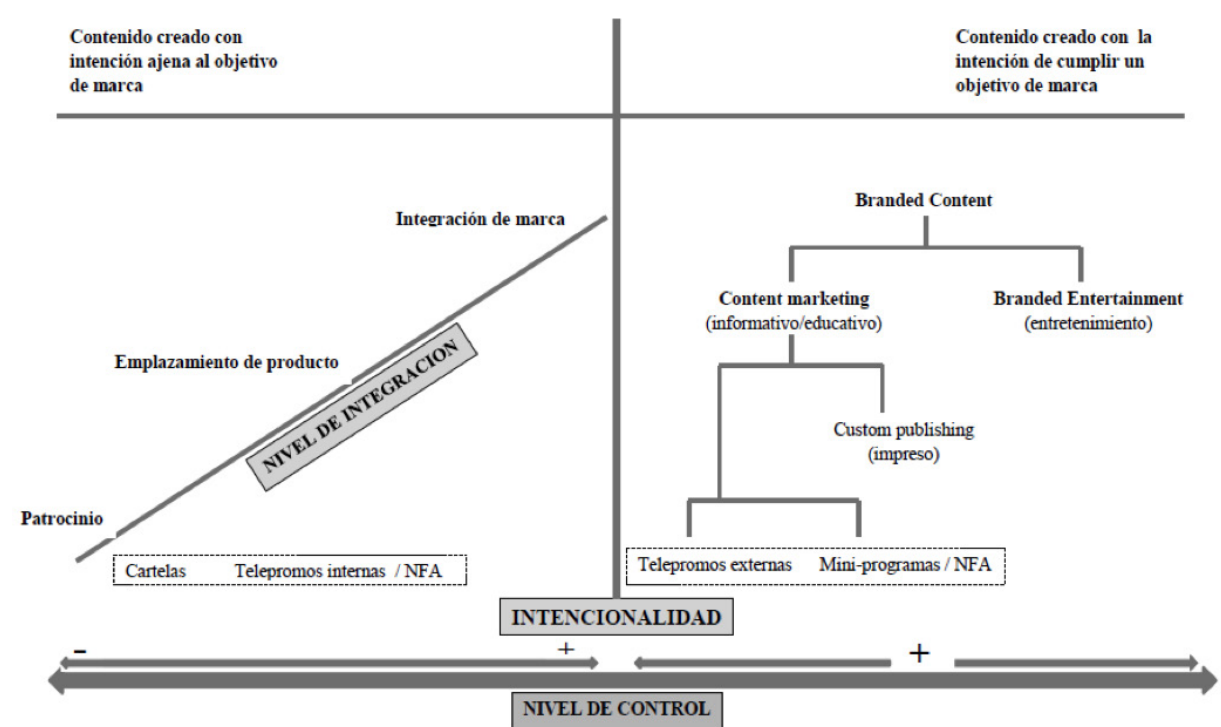

Figura 2: Clasificación de los mensajes híbridos (Fuente: Aguilera, 2014)

\section{Conclusiones}

Los mensajes híbridos combinan un mensaje comercial con contenido no-comercial. Desde que Balasubramanian identificó este tipo de mensajes y estableció una primera clasificación, la variedad de mensajes híbridos se ha multiplicado, especialmente con la revolución digital, lo que provoca una gran confusión tanto en la definición como en la clasificación de este tipo de mensajes.

En una primera aproximación a una clasificación de los mensajes híbridos encontramos como elementos diferenciadores: lo explícito o implícito de la presencia de la marca, el nivel de control y el nivel de integración.

Sin embargo, nosotros defendemos que el criterio de diferenciación más importante en este tipo de mensajes es la intencionalidad del emisor en la creación del contenido, clasificándolos en dos grandes grupos: 
Los Mensajes Híbridos en el marketing postmoderno: una propuesta de taxonomía | 54

- Mensajes híbridos resultado de incorporar una marca dentro de un contenido creado con fines ajenos al de la marca insertada. Dentro de esta categoría, los mensajes se diferenciarían por el nivel de integración.

- Mensajes híbridos cuyo objetivo es transmitir un mensaje de marca, dotándole de suficiente interés como para atraer a su público objetivo. Dentro de esta categoría la principal diferencia es el tipo de contenido: entretenimiento o informativo.

Nuestra propuesta también considera que el branded entertainment y el content marketing son dos sub-formas de branded content. El primero es la forma que adopta el branded content cuando es entretenimiento, mientras que el content marketing es informativo/educativo.

\section{Referencias}

Aguilera, J. de (2014). Branded Entertainment. Los contenidos de marca como herramienta de comunicación comercial en el entorno del marketing actual. (Tesis inédita de doctorado). Universidad Rey Juan Carlos.

Balasubramanian, S. K. (1994). Beyond Advertising and Publicity: Hybrid Messages and Public Policy issues. Journal of Advertising, 23 (4), 29-46.

Baños, M. y Rodríguez, T. (2003). Product Placement. Estrella invitada: la marca. Madrid: CIE Dossat.

Branded Content Marketing Association (2014). www.thebcma.info

Canter, A, Asmussen, B, Michels, N., Butler, A. y Thomson, S. (2013). Defining Branded Content for the Digital Age. En Developing a clearer understanding of a 'new' key marketing concept. 0xford: 0xford Brookes University.

Content Marketing Asociation (2014). www.the-cma.com

Content Marketing Institute (2012). Your kick-start guide to engaging content. Recuperado de http://www.slideshare.net/CMI/your-kickstart-guide-to-engaging-content

D'Astous, A. y Séguin, N. (1999). Consumer reactions to product placement strategies in television sponsorship. European Journal of Marketing, 33 (9/10), 896-910. D0I: 10.1108/03090569910285832 
55 | Joaquín de Aguilera Moyano, Miguel Baños González y Francisco Javier Ramírez Perdiguero

INNOVACIÓN TEÓRICA

Duncan, T. (2012, 28 de noviembre). Branding and Content: finding the right balance - A Content Marketing whitepaper. Studio One. Recuperado de http:// contentmarketinginstitute.com/2012/02/history-content-marketinginfographic/ Entertainment and Resources Marketing Association (2014). http://erma.org/ Gambetti, R. C. y Graffigna, G. (2010). The concept of engagement: a systematic análisis of the ongoing marketing debate. International Journal of Market Research, 52(6), 801- 826. D0I:10.2501/S1470785310201661

Hudson, S. y Hudson, D. (2006).Branded Entertainment: a new Advertising technique or Product Placement in disguise. Journal of Marketing Management, 22, 489-504. DOI: 10.1362/026725706777978703.

Keller, K. L. (2008). Strategic Brand Management. Building, measuring and managing Brand Equity. Nueva Jersey: Pearson Prentice Hall.

Kotler, P. (2005). Foreword. En Tybout, A. M. y Calkins, T. (Eds.) Kellogg on Branding. Hoboken: Wiley.

Lehu, J.M. (2007). Branded entertainment: product placement and brand strategy in the entertainment business. London: Kogan Page.

Lieb, R., Silva, C. y Tran, C. (2013, 25 de abril). Organizing for Content: models to incorporate Content Strategy and Content Marketing in the Enterprise. Recuperado de http://www.altimetergroup.com/research/reports/organizing-for-content

Martí, J. (2010). Funny marketing: consumidores, entretenimiento y comunicaciones de marketing en la era del branded entertainment. Madrid: Volkers-Kluwer.

Mayar, V. y Ramsey, G. (2011). Digital impact. The two secrets to online marketing success. Hoboken: John Wiley \& Sons.

Neurads (Agencia Audiovisual de Contenidos Creativos). (2013) ¿Qué diferencia al Product Placement del Branded Content? Recuperado de http://www.neurads. com/product-placement-vs-branded-content/

Nosrati, M., Karimi, R, Mohamadi, M. y Maleikian, K. (2013). Internet Marketing or modern Advertising! How? Why? International Journal of Economy, Management and Social Sciences, 2 (3), 56-63.

Pino, C. del, Olivares, F. (2007). Brand Placement y Advertainment: integración y fusión entra la ficción audiovisual y las marcas. Zer, 22, 341-367.

Pulizzi, J. (2012). The transformation of Content Marketing. EContent Magazine, 35(10), 20-21. Recuperado de http://www.econtentmag.com/Articles/ Editorial/Commentary/The-Transformation-of-Content-Marketing-86389.htm

ICONO14 | Año 2016 Volumen $14 \mathrm{~N}^{\circ} 1$ | ISSN: 1697-8293 | DOI: ri14.v14i1.890 
Los Mensajes Híbridos en el marketing postmoderno: una propuesta de taxonomía | 56

INNOVACIÓN TEÓRICA

Rahim, K. y Clemens, B. (2012). Organizational goals and performance measurement for Content Marketing. Journal of Communication and Computer, 9, 896-904.

Ramos, R. (2013). Content Marketing. Insider's secret to online sales \& lead generation. Nueva York: One Night Expert Publishing.

Ramos, M. (2006). Cuando la marca ofrece entretenimiento: aproximación al concepto de advertainment. Questiones Publicitarias, I(11),33-49.

Rappaport, S.D. (2007).Lessons from online practice: new advertising models. Journal of Advertising Research, 47(2), 135-141. D0I:10.2501/S0021849907070158

Regueira, F. J. (2012). El contenido como herramienta eficaz de comunicación e marca. Análisis teórico y empírico (tesis doctoral), Departamento de Economía de la Empresa, Universidad Rey Juan Carlos. Recuperado de http://hdl.handle. net/10115/11415

Reinares, P. y Reinares, R. (2013). Are the new forms of television advertising beneficial for the advertisers and the TV management? A Spanish television advertising study. International Journal on Media Management, 15:3, 161-175. DOI: $10.1080 / 14241277.2013 .767260$

Rose, R. y Pulizzi, J. (2011). Managing Content Marketing. The real-world guide for creating passionate subscribers to your brand. Cleveland: CMI Books.

Russell, C. A. (2007). Advertainment: fusing advertisement and entertainment. Yaffe Center for Persuasive Communication, University of Michigan. Recuperado de http://www.yaffecenter.org/downloads/Advertainment_teaching_materials. pdf

Sheehan, K. B. y Guo, A. (2005). Leaving on a (branded) jet plane: an exploration of audience attitudes towards product assimilation in television content. Journal of Current Issues in Advertising, 27 (1), 79-91. D0I: 10.1080/10641734.2005.10505175

Soares Negrao, M. (2011). Product Placement and Branded Entertainment: A look into the alternative advertising methods. (trabajo para Master Degree Report), Graduate School of the University of Texas at Austin

The Content Council (2014). http://thecontentcouncil.org/

Tuomi, C. (2010). Branded Entertainment. Discovering the Possibilities in Mobile Media. Master's Thesis, Hanken School of Economics, Department of Marketing. Recuperado de https://helda.helsinki.fi/bitstream/handle/10227/532/tuomi. pdf?sequence $=1$

DOI: ri14.v14i1.890 | ISSN: 1697-8293 | Año 2016 Volumen 14 Nº 1 | ICONO14 
57 | Joaquín de Aguilera Moyano, Miguel Baños González y Francisco Javier Ramírez Perdiguero

INNOVACIÓN TEÓRICA

Valiquette, M. (2013, 17 de julio). The gap between ads and branded content. Marketing Magazine. Recuperado de http://www.marketingmag.ca/ uncategorized/column-the-gapbetween-ads-and-branded-content-83757

Walton, A. (2010). The evolution of Product Placement in film. The Elon Journal of Undergraduate Research in Communications, 1(1), 76-85.

Williams, K., Petrosky, A., Hernandez, E. y Page, R. (2011). Product Placement effectiveness: revisited and renewed. Journal of Management and Marketing Research ,7(1),1-24. Recuperado dehttp://www.aabri.com/manuscripts/10712. pdf

Zmuda, N. (2013, 14 de enero). Just what is Content Marketing? Advertising Age, 84(2), 13.

Zyman, S. (1999). The end of Marketing as we know it. Nueva York: Harper Collins. 